\title{
Left Atrial Enlargement on Non-Gated CT Is Associated with Large Vessel Occlusion in Acute Ischaemic Stroke
}

\author{
Waleed Butt ${ }^{a}$ Permesh Singh Dhillon ${ }^{a}$ Robert Lenthall ${ }^{a}$ Luqman Malik $^{a}$ \\ Wazim Izzath $^{\mathrm{a}}$ Kailash Krishnan $^{\mathrm{b}}$ Bindu George $^{c}$ Kate Pointon $^{\mathrm{c}}$ \\ anterventional Neuroradiology Department, Queen's Medical Centre, Nottingham University Hospitals NHS \\ Trust, Nottingham, UK; b Stroke Medicine, Nottingham City Hospital, Nottingham University Hospitals NHS Trust, \\ Nottingham, UK; ' Cardiothoracic Radiology Department, Nottingham City Hospital, Nottingham University \\ Hospitals NHS Trust, Nottingham, UK
}

\section{Keywords}

Stroke · Atrial fibrillation · Atrial enlargement · Large vessel occlusion

\begin{abstract}
Background: Recent reports have suggested that atrial fibrillation (AF) is more prevalent in the large vessel occlusion (LVO) subgroup of acute ischaemic stroke patients. Given the association between left atrial enlargement (LAE) and $A F$, we sought to evaluate the feasibility of assessing LAE on non-gated CT and its association with LVO in the hyperacute stroke setting. Methods: We analysed our prospectively collected database that included all stroke patients referred for consideration of endovascular treatment between April 14, 2020, and May 21, 2020. During this period, a CT chest was included in our regional stroke protocol to aid triage of patients suspected for COVID-19 from which cardiac measurements were obtained. Patients were dichotomized into LVO and no-LVO groups, and LA measurements were trichotomized into normal, borderline, and enlarged. Univariate analyses were performed between groups. Results: Of the included 38 patients, 21 were categorized as LVO and 17 as no LVO. There was a statistically significant association be-
\end{abstract}

karger@karger.com www.karger.com/cee

Karger"

GOPEN ACCESS
(C) 2021 The Author(s)

Published by S. Karger AG, Basel

This is an Open Access article licensed under the Creative Commons Attribution-NonCommercial-4.0 International License (CC BY-NC) (http://www.karger.com/Services/OpenAccessLicense), applicable to the online version of the article only. Usage and distribution for commercial purposes requires written permission. tween LAE and LVO ( $p=0.028)$. No significant difference was demonstrated between groups for the baseline AF and other clinical characteristics, except for baseline NIHSS ( $p=$ $0.0005)$. There was excellent inter- and intra-rater reliability (ICC $=0.969)$ for LA measurements. Conclusion: Our study provides preliminary data to suggest LAE is more prevalent in the LVO stroke subgroup at presentation and can be reliably assessed on non-gated CT in the hyperacute setting. These findings have potential implications for stratifying secondary management and may prompt a more rigorous pursuit of occult AF or other cardiac causes of stroke.

(c) 2021 The Author(s)

Published by S. Karger AG, Basel

\section{Introduction}

Large vessel occlusion (LVO) of the proximal intracranial circulation may account for $>30 \%$ of all acute ischaemic stroke (AIS) presentations and without treatment is associated with significantly worse outcomes compared to non-LVO AIS $[1,2]$. The aetiology of LVO can be due to (1) in situ thrombosis of the primary arterial site secondary to underlying atherosclerosis, (2) artery-to-artery embolus from an extra-cranial lesion such as an ath- 
erosclerotic plaque or dissection, (3) cardiac embolism, or (4) cryptogenic causes of vessel occlusion. Cardioembolism accounts for an increasing share of AIS with the most common risk factor being atrial fibrillation (AF) [3].

Recent studies have suggested that AF is more common in the LVO subgroup stroke population [4-6]. Given the association between left atrial enlargement (LAE) and AF $[7,8]$, we hypothesized LAE may be associated with LVO and thereby indicate potential stroke aetiology and direct secondary management. Cardiac imaging is not routinely performed in the hyperacute stroke setting, and there is variation in patient selection for subsequent echocardiographic assessment, which is limited in availability and is operator dependent [9].

The mandate to detect coronavirus disease 2019 (COVID-19) led to the inclusion of thoracic CT in our regional stroke imaging protocol to aid risk stratification for patients and clinical staff. Acquisition of thoracic CT also provided a basic cardiac imaging dataset, acquired at presentation in ischaemic stroke patients deemed clinically eligible for EVT by our referring centres. Herein, we sought to evaluate LAE on non-ECG-gated CT in patients with and without angiographic evidence of LVO in this cohort.

\section{Methods}

This study was registered and approved by our institution's audit committee, and individual patient consent was waived. We reviewed our prospectively collated database of all stroke referrals considered for EVT between April 14, 2020, and May 21, 2020. Additional clinical variables were obtained from the electronic hospital records.

In our standard regional imaging algorithm, all acute stroke referrals presenting with a suspected LVO within $24 \mathrm{~h}$ of symptom onset undergo an unenhanced CT head and dual-phase CT angiogram from the aortic arch to the skull vertex. As previously described in our recent publication [10], our modified protocol included the addition of a non-contrast or delayed postcontrast chest CT for all patients except those who had a confirmed COVID-19 status. Data collated included patient demographics, presence of LVO, tandem carotid or vertebral lesion, LA measurement, AF, hypertension, diabetes mellitus, cardiac failure, previous stroke or transient ischaemic attack, baseline National Institutes of Health Stroke Scale (NIHSS), COVID-19 status, and anticoagulant drug status at presentation.

\section{Chest CT Parameters}

Non-ECG-gated chest CT images were obtained on a Somatom Definition AS or AS Plus, 64- or 128-slice multidetector CT system (Siemens Healthineers, Camberley, UK) at our institution. The scan range was from the lung apices to the diaphragms immediately following acquisition of the dual-phase CT angiogram from the aortic arch to the skull vertex. The following acquisition pa-

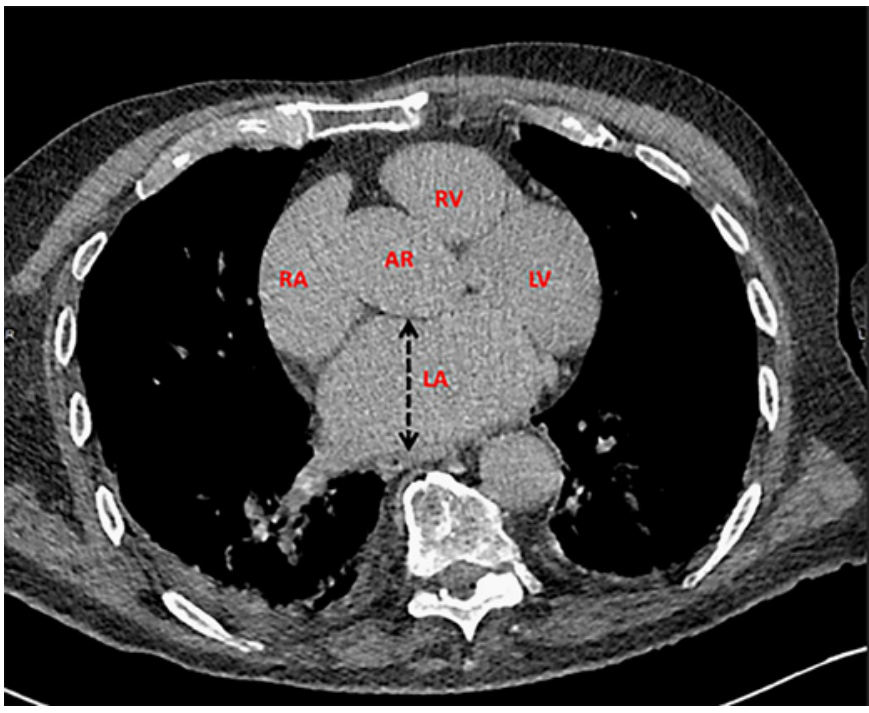

Fig. 1. Axial non-ECG-gated CT at the level of the aortic root, demonstrating an enlarged left atrium. The left atrial diameter is measured in the maximal anterior-posterior dimension (black dotted arrow). LA, left atrium; RA, right atrium; AR, aortic root; LV, left ventricle; $R V$, right ventricle; $C T$, computed tomography.

rameters were used: tube voltage of $120 \mathrm{kV}$, tube current of $70 \mathrm{~mA}$, 0.5 -s helical rotation time, and 1.2 helical pitch. The reconstructed axial CT slice thickness was $1 \mathrm{~mm}$, and further sections were reconstructed in the axial, coronal, and sagittal planes of $2-\mathrm{mm}$ section thickness at $2-\mathrm{mm}$ increments. The effective radiation dose calculated from the total dose length product and additional time incurred from the completion of the dual-phase CT angiogram to the completion of CT chest were obtained from the radiological information system. There were variations of the scanner models and acquisition parameters across the regional hospitals.

\section{Imaging Analysis}

Craniocervical imaging was reviewed by a neuroradiologist whilst the cardiac findings were analysed and recorded by 2 independent cardiothoracic radiologists who were blinded to the neurovascular findings. LVO was defined by occlusion of the internal cerebral artery, M1/M2 middle cerebral artery, or the basilar artery. The LA measurement was taken in the anterior-posterior direction on non-reformatted axial images at the level of the aortic root (Fig. 1). LA measurement was trichotomized into normal $(<43 \mathrm{~mm})$, borderline (43-47 mm), and enlarged $(>47 \mathrm{~mm})$.

\section{Statistical Analysis}

Standard data and statistical analysis (including $t$ test, MannWhitney U test, and $\chi^{2}$ or Fisher's exact test) was carried out using GraphPad Prism (GraphPad Software, Inc.). Statistical significance was set at $p<0.05$. Comparisons were made using the LA measurements as a continuous variable and LAE classed as a categorical variable. Univariate analyses were performed between groups. Intra-class correlation coefficients (ICCs) were calculated as a measure of inter-rater (2-way random effect, absolute agreement) and intra-rater (2-way mixed effect, absolute agreement) reliability between 2 cardiothoracic radiologists using the LA mea- 
Table 1. Clinical characteristics in the LVO and no-LVO groups
Fig. 2. Study flowchart with patients dichotomized into LVO and no-LVO. LA size categorized as normal $(<43 \mathrm{~mm})$, borderline (43-47 $\mathrm{mm})$, and enlarged $(>47$ $\mathrm{mm})$. LA, left atrial; LVO, large vessel occlusion; EVT, endovascular treatment; CT, computed tomography.

\begin{tabular}{llll}
\hline Characteristics & LVO $(n=21)$ & No LVO $(n=17)$ & $p$ value \\
\hline Mean age, years (SD) & $75.3(10.7)$ & $71.1(14.4)$ & 0.309 \\
Male sex, $n$ (\%) & $10(47)$ & $9(53)$ & 1 \\
Baseline NIHSS & $16.5(7.9)$ & $4.5(1.8)$ & $\mathbf{0 . 0 0 0 5}$ \\
Hypertension & 8 & 5 & 0.734 \\
Diabetes mellitus & 2 & 2 & 1 \\
Atrial fibrillation & 6 & 2 & 0.257 \\
Anticoagulants & 4 & 0 & 0.113 \\
Previous history of stroke/TIA & 3 & 2 & 1 \\
COVID-19 confirmed & 1 & 1 & 1 \\
Median LA diameter, mm (IQR) & $46(41-53)$ & $41(31.2-43.5)$ & $\mathbf{0 . 0 2 8}$ \\
\hline
\end{tabular}

LVO, large vessel occlusion; LA, left atrium; TIA, transient ischaemic attack; COVID-19, coronavirus disease 2019 confirmed by RT-PCR; NIHSS, National Institutes of Health Stroke Scale.

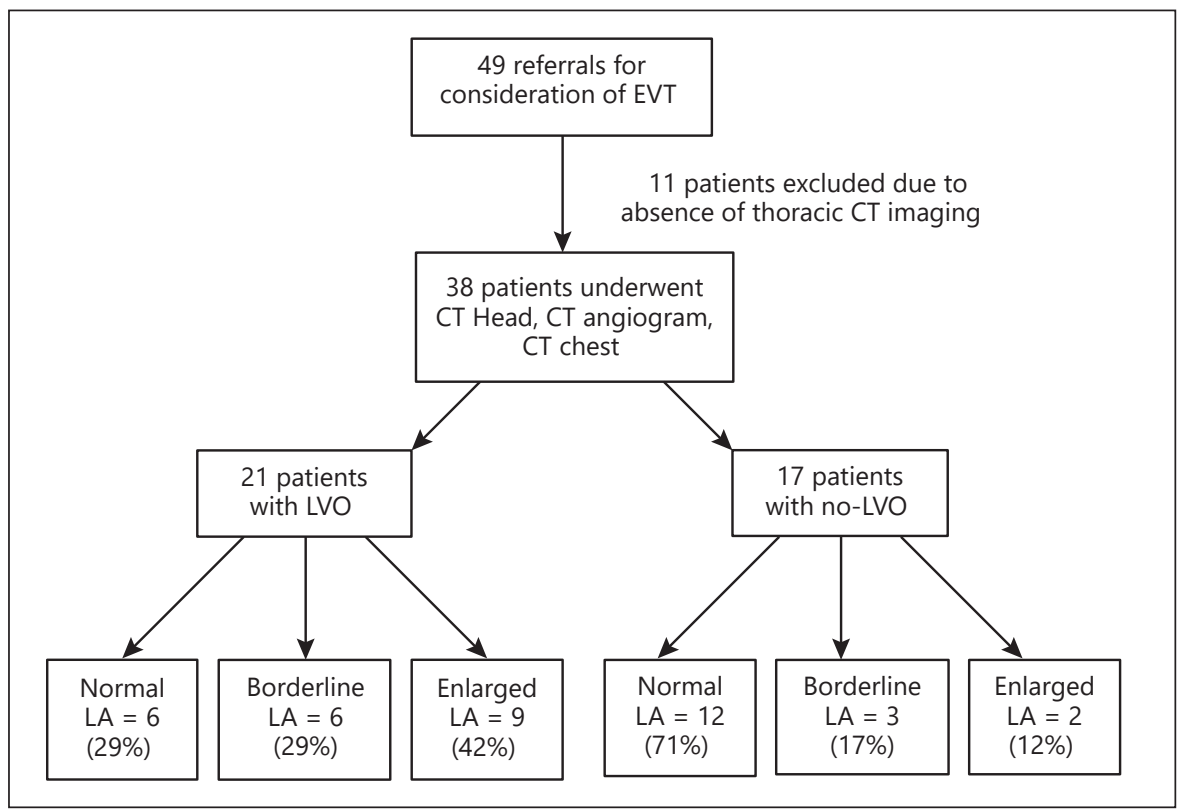

surements in a randomly generated sample of 18 patients of the study population. Both raters were blinded to the neurovascular findings and primary LA measurements. The second LA measurement readings of the primary rater were performed following a 2 -week interval for the intra-rater reliability. ICC $>0.75=$ excellent reliability, $0.60-0.74=$ good reliability, $0.41-0.59=$ fair reliability, and $<0.40=$ poor reliability.

\section{Results}

Since the implementation of the revised imaging protocol during the study period, we received 49 referrals for consideration of EVT. Thoracic CT imaging was not performed in 11 patients, and hence these patients were ex- cluded from the final analysis. Of the included 38 patients, the mean age was $72.9 \pm 12.6(\mathrm{SD})$ years, and there were 19 males and 19 females. The mean additional time incurred for the chest CT was $184 \pm 65.5$ (SD) s, range of $45-337 \mathrm{~s}$. The average added effective radiation dose from the extension of the chest CT was $2.47 \pm 1.03$ (SD) $\mathrm{mSv}$. No patient was identified to have a tandem lesion.

The patient characteristics, dichotomized into 2 groups (21 LVO vs. 17 no LVO), are summarized in (Table 1). There was a statistically significant association between LA size categories and the presence of LVO $(p=$ 0.028), which is presented in Figure 2. The median LA measurements were $46 \mathrm{~mm}$ (IQR 41-53) and $41 \mathrm{~mm}$ (IQR 31.2-43.5) in the LVO and no-LVO groups, respec- 
tively $(p=0.028)$. Except for the baseline NIHSS scores ( $p=0.0005)$, no significant difference was demonstrated between groups for the age, sex, AF, hypertension, diabetes, cardiac failure (none in each group), previous stroke or transient ischaemic attack, COVID-19 status, and anticoagulation use. There was excellent inter-rater and intra-rater reliability (ICC $=0.969$ [95\% CI: 0.917-0.988; $p=0.0001]$ and ICC $=0.979$ [95\% CI: 0.946-0.992; $p=$ $0.0001])$, respectively.

\section{Discussion}

Our results indicate an association between LAE demonstrated on non-ECG-gated CT and LVO. The implication of the increased frequency of LVO in the LAE group in comparison with the borderline group remains unclear. However, it is plausible that only "moderate" or "severe" LAE is associated with LVO. This would be consistent with previous findings in which the degree of LAE is related to the cumulative risk of adverse cardiovascular outcomes including heart failure, AF, myocardial infarction, and stroke [11].

Chang et al. [12] investigated cardiac markers for LVO and reported an association between LVO and LA diameter on transthoracic echocardiography (TTE); however, an association with LAE could not be gleaned from their study. Furthermore, whilst TTE remains the most widely used modality to assess LA size, it is operator dependent, time intensive, and may have technical limitations [13]. In practice, TTE is not always rapidly or consistently utilized, which in turn may cause a delay to diagnosis.

Modern multidetector CT that is widely available has capabilities that enable rapid image acquisition to mitigate cardiac motion artefacts with enhanced temporal and spatial resolution. It has been shown that using a 43$\mathrm{mm}$ cutoff on non-ECG-gated CT for the greatest anterior-posterior LA diameter on axial non-reformatted images provides $60.5 \%$ specificity and $85 \%$ sensitivity when compared with echocardiographic dimensions [14]. When using a threshold of $45 \mathrm{~mm}$, the specificity has been reported as 94\% [15]. Given the threshold-dependent trade-off between sensitivity and specificity, we took the view that using a single cutoff value to dichotomize LAE would limit accuracy and fail to account for errors in measurement due to non-contrast acquisition and interobserver variation which in turn may mask a true association.

There are emerging prospective data to suggest that in the cryptogenic stroke population, LVO is independently associated with covert paroxysmal AF [16]. There is also a higher incidence of cardioembolic aetiology in LVO recurrence [17]. Although risk factors for ischaemic stroke are well known, the cause remains undefined in 25-39\% of patients who experience an acute event, even with full diagnostic evaluation [18]. In the recent secondary analysis of the NAVIGATE ESUS trial, it was shown that in patients with embolic stroke of undetermined source and LAE, treatment with rivaroxaban resulted in a $74 \%$ reduction in stroke recurrence [19]. Our findings therefore have potential implications as the increased presence of LAE in the LVO subgroup may prompt a more rigorous pursuit of occult AF or other cardiac causes of stroke and aid secondary prevention stratification in future trials assessing LAE as a treatment target. An association between $\mathrm{AF}$ and LVO was not revealed in our study, and this may have been due to underinvestigation or sample size.

Imaging algorithms for ischaemic stroke do not routinely include cardiac imaging in the hyperacute period, although the feasibility has been demonstrated without incurring a time penalty in a "triple-rule-out" protocol [20] and in the present study. Furthermore, the mean radiation dose of $2.47 \mathrm{mSv}$ for the chest CT we report falls below the average radiation dose per person/annum in the UK. LA measurements proved to be simple, quick, and reproducible, demonstrated by the excellent intraand inter-rater agreement.

Although the baseline data were prospectively collated, our study is limited by its single-centre retrospective review and sample size. Second, our population may have excluded patients ineligible for EVT on clinical grounds (modified Rankin score $>2$ and NIHSS $<5$ ), which limits the generalizability of our findings. Third, it is not possible to establish causality as not all clinical factors, including body surface area, that influence LA size were analysed. Last, the disproportionate number of patients with LVO who were excluded due to the absence of thoracic imaging (10 out of 11 ) may introduce a sampling bias.

\section{Conclusion}

Our study of a patient cohort presenting during the COVID-19 pandemic demonstrates feasibility of the inclusion of non-ECG-gated cardiac CT imaging during the immediate workup of acute stroke presentation and provides preliminary evidence that LAE may be associated with LVO. The presence of LAE in LVO may prompt a more thorough workup and management of an underly-
Cerebrovasc Dis Extra 2021;11:87-91 DOI: $10.1159 / 000519121$
Butt/Dhillon/Lenthall/Malik/Izzath/ Krishnan/George/Pointon 
ing cardiogenic cause to prevent stroke recurrence. Larger studies and prospective validation are warranted prior to clinical application.

\section{Statement of Ethics}

Ethical statement is not required.

\section{Conflict of Interest Statement}

The authors have no conflicts of interest to declare.

\section{Author Contributions}

W.B., P.S.D., and R.L. contributed to study conception and design. W.B., P.S.D., L.M., B.G., and K.P. contributed to acquisition of the data. W.B., P.S.D., B.G., R.L., and K.P. contributed to analysis and interpretation of the data. K.K., W.I., L.M., and R.L. contributed to critical revision of the manuscript. R.L. and K.P. contributed to study supervision.

\section{Data Availability Statement}

All data generated or analysed during this study are included in this article. Further enquiries can be directed to the corresponding author.

\section{Funding Sources}

The authors received no funding for this study.

\section{References}

1 Malhotra K, Gornbein J, Saver JL. Ischemic strokes due to large-vessel occlusions contribute disproportionately to stroke-related dependence and death: a review. Front Neurol. 2017;8:651.

2 Lakomkin N, Dhamoon M, Carroll K, Singh IP, Tuhrim S, Lee J, et al. Prevalence of large vessel occlusion in patients presenting with acute ischemic stroke: a 10-year systematic review of the literature. J Neurointerv Surg. 2019;11(3):241-5.

3 Yiin GS, Howard DP, Paul NL, Li L, LuengoFernandez R, Bull LM, et al. Age-specific incidence, outcome, cost, and projected future burden of atrial fibrillation-related embolic vascular events: a population-based study. Circulation. 2014;130(15):1236-44.

4 Inoue M, Noda R, Yamaguchi S, Tamai Y, Miyahara M, Yanagisawa S, et al. Specific factors to predict large-vessel occlusion in acute stroke patients. J Stroke Cerebrovasc Dis. 2018;27(4):886-91.

5 Grewal P, Lahoti S, Aroor S, Snyder K, Pettigrew LC, Goldstein LB. Effect of known atrial fibrillation and anticoagulation status on the prehospital identification of large vessel occlusion. J Stroke Cerebrovasc Dis. 2019; 28(12): 104404

6 Seachrist E, Jacob S, Ferari C, Ranasinghe T, Petrone A, Adcock A. How much does atrial fibrillation contribute to large vessel occlusions? (P1.3-049). Neurology. 2019;92(15 Suppl).

7 Sanfilippo AJ, Abascal VM, Sheehan M, Oertel LB, Harrigan P, Hughes RA, et al. Atrial enlargement as a consequence of atrial fibrillation. A prospective echocardiographic study. Circulation. 1990;82(3):792-7.
8 Seko Y, Kato T, Haruna T, Izumi T, Miyamoto S, Nakane E, et al. Association between atrial fibrillation, atrial enlargement, and left ventricular geometric remodeling. Sci Rep. 2018; 8(1):6366

9 Morris JG, Duffis EJ, Fisher M. Cardiac workup of ischemic stroke: can we improve our diagnostic yield? Stroke. 2009;40(8):2893-8.

10 Dhillon PS, Pointon K, Lenthall R, Nair S, Subramanian G, McConachie N, et al. Regional mechanical thrombectomy imaging protocol in patients presenting with acute ischaemic stroke during the COVID-19 pandemic. AJNR Am J Neuroradiol. 2020;41(10):1849-55.

11 Abhayaratna WP, Seward JB, Appleton CP, Douglas PS, Oh JK, Tajik AJ, et al. Left atrial size: physiologic determinants and clinical applications. J Am Coll Cardiol. 2006;47(12): 2357-63.

12 Chang A, Ricci B, Grory BM, Cutting S, Burton T, Dakay K, et al. Cardiac biomarkers predict large vessel occlusion in patients with ischemic stroke. J Stroke Cerebrovasc Dis. 2019;28(6):1726-31.

13 Malik SB, Chen N, Parker RA 3rd, Hsu JY. Transthoracic echocardiography: pitfalls and limitations as delineated at cardiac CT and MR imaging. Radiographics. 2017;37(2):383-406.

14 Sohrabi S, Hope M, Saloner D, Keedy A, Naeger D, Lorca MC, et al. Left atrial transverse diameter on computed tomography angiography can accurately diagnose left atrial enlargement in patients with atrial fibrillation. J Thorac Imaging. 2015;30(3):214-7.
15 Huckleberry J, Haltom S, Issac T, Gabaldon J, Ketai L. Accuracy of non-ECG-gated computed tomography angiography of the chest in assessment of left-sided cardiac chamber enlargement. J Thorac Imaging. 2012;27(6): 354-8.

16 Pagola J, Juega J, Francisco-Pascual J, Bustamante A, Penalba A, Pala E, et al. Large vessel occlusion is independently associated with atrial fibrillation detection. Eur J Neurol. 2020;27(8):1618-24.

17 Styczen H, Maegerlein C, Yeo LL, Clajus C, Kastrup A, Abdullayev N, et al. Repeated mechanical thrombectomy in short-term large vessel occlusion recurrence: multicenter study and systematic review of the literature. J Neurointerv Surg. 2020;12:118693.

18 Amarenco P. Underlying pathology of stroke of unknown cause (cryptogenic stroke). Cerebrovasc Dis. 2009;27(Suppl 1): 97-103.

19 Healey JS, Gladstone DJ, Swaminathan B, Eckstein J, Mundl H, Epstein AE, et al. Recurrent stroke with rivaroxaban compared with aspirin according to predictors of atrial fibrillation: secondary analysis of the NAVIGATE ESUS randomized clinical trial. JAMA Neurol. 2019;76(7):764-73.

20 Furtado AD, Adraktas DD, Brasic N, Cheng SC, Ordovas K, Smith WS, et al. The triple rule-out for acute ischemic stroke: imaging the brain, carotid arteries, aorta, and heart. AJNR Am J Neuroradiol. 2010;31(7):1290-6. 